\title{
Composição química do óleo volátil de Myrcianthes nativas da região sul do Brasil
}

\author{
Miriam A. Apel*, Marcos Sobral, Amélia T. Henriques \\ ${ }^{1}$ Faculdade de Farmácia, Universidade Federal do Rio Grande do Sul, Av. Ipiranga 2752, \\ 90610-000, Porto Alegre, RS, Brasil
}

\begin{abstract}
RESUMO: O óleo essencial das folhas de Myrcianthes gigantea (Myrtaceae), coletadas no Rio Grande do Sul, foi obtido por hidrodestilação em Clevenger e analisado por CG/detector de ionização de chamas e CG/EM. Trinta e seis compostos foram identificados, totalizando 90,1\% do conteúdo do óleo. A composição do óleo demonstrou predominância de sesquiterpenos cíclicos, principalmente da via de ciclização do germacrano, apresentando espatulenol $(28,8 \%)$ e seu isômero, iso-espatulenol (9,5\%), como principais constituintes. A composição do óleo das folhas de M. cisplatensis e $M$. pungens, coletadas na mesma região, também foi analisada e comparada com estudos prévios reportados para estas espécies coletadas em outros países da América da Sul. O óleo essencial de $M$. cisplatensis apresentou um alto conteúdo de monoterpenos (56,3\%), especialmente das séries pinano e $p$-mentano, com três compostos majoritários: 1,8-cineol $(29,8 \%)$, limoneno $(10,9 \%)$ e $\alpha$-pineno $(8,9 \%)$, sendo similar ao reportado. Para o óleo de $M$. pungens 36 compostos foram identificados, porém sem predomínio majoritário, onde $\beta$-cariofileno $(10,1 \%)$, foi o principal deles. O óleo desta espécie difere do relatado para exemplares coletados na Argentina, rico em monoterpenos, sugerindo uma possível ocorrência de quimiotipos.
\end{abstract}

Unitermos: Myrcianthes gigantea, Myrcianthes pungens, Myrcianthes cisplatensis, Myrtaceae, óleos essenciais, espatulenol, 1,8-cineol, limoneno.

\begin{abstract}
Chemical composition of the volatile oils of native Myrcianthes species from South Brazil". The essential oil of Myrcianthes gigantea (Myrtaceae) leaves, which were collected in Rio Grande do Sul (Southern Brazil), was obtained by hydrodistillation in a Clevenger apparatus. The chemical investigation was performed by GC-FID and GC-MS. Thirty-six compounds were identified accounting for $90.1 \%$ of the oil content. The analyzed species was rich in cyclic sesquiterpenes, mainly those from the germecrane cyclization pathway, among them spathulenol (28.8\%) and its isomer, iso-spathulenol (9.5\%). Leaves of M. cisplatensis and M. pungens were also collected in the same region and the analysis of the oil composition was compared with those previously reported for collections from other countries in South America. M. cisplatensis oil showed a high content of monoterpenes $(56.3 \%)$, specially from the $p$-mentane and pinane groups, with three major compounds: 1,8 -cineole $(29.8 \%)$, limonene $(10.9 \%)$ and $\alpha$-pinene $(8.9 \%)$. These findings are in agreement with those reported previously. For M. pungens, thirty-six compounds were identified without a predominant component. $\beta$-caryophyllene $(10.1 \%)$, with caryophyllane skeleton, was the main substance for this sample. For this species the oil composition was quite different from those related for two exemplars collected in Argentina, in which both oil were rich in monoterpenes. This result suggests the occurrence of chemotypes.
\end{abstract}

Keywords: Myrcianthes gigantea, Myrcianthes pungens, Myrcianthes cisplatensis, Myrtaceae, essential oil composition, spathulenol, 1,8-cineole, limonene.

\section{INTRODUÇÃO}

$\mathrm{O}$ gênero Myrcianthes pertence à família Myrtaceae, e apresenta cerca de 30 espécies (Grifo, 1992), a maior parte distribuída na América do Sul, região dos Andes, com ocorrência de quatro delas no Sul do Brasil. Três destas são nativas do Rio Grande do Sul.

Myrcianthes cisplatensis (Cambess.) O. Berg (nome popular: murta) é uma árvore pequena (até $5 \mathrm{~m}$ ) e ocorre no Uruguai, Argentina e Sul do Brasil (Marchiori; Sobral, 1997). Popularmente é utilizada especialmente para lavagem e cicatrização de feridas na pele além de ser considerado um bom remédio para afecções brônquicas e dos pulmões (Font Quer, 1988). Estudos demonstraram que o extrato aquoso desta espécie apresenta ação antiviral contra o vírus respiratório RSV (Kott et al., 1999). Em recente estudo envolvendo atividade antimicrobiana dos extratos aquoso, alcoólico e hidroalcoólico nenhuma resposta positiva foi observada, quando testado frente a um painel de bactérias Gram positivas e negativas e de fungos (Penna et al., 2005).

M. gigantea (D. Legrand) D. Legrand (nome popular: araçá-do-mato) é uma árvore de até $15 \mathrm{~m}$ de altura e ocorre desde o norte do Uruguai até o nordeste 
do Brasil (Marchiori; Sobral, 1997). Não foi encontrado relato de uso popular.

M. pungens (O. Berg) D. Legrand (nome popular: guabijú) é uma árvore de porte médio a grande, de até $25 \mathrm{~m}$ de altura distribuída nas florestas desde o estado de São Paulo até o norte do Uruguai, alcançando a Bolívia, Argentina e Paraguai (Marchiori; Sobral, 1997). Na medicina popular, esta espécie é utilizada por suas propriedades antidiarréicas (Pio Corrêa, 1984).

Estudos anteriores envolvendo a análise do óleo volátil de espécies de Myrcianthes coletadas em diversos países da América do Sul são reportados. Carmen e colaboradores (1972) caracterizaram o óleo de $M$. callicoma pela presença de $\alpha$-pineno, limoneno e 1,8-cineol; $M$. fragrans, coletada na Jamaica e em Cuba apresentou como compostos majoritários limoneno e $\alpha$ terpineol e limoneno e $\alpha$-pineno, respectivamente (Tucker et al., 1992; Pino et al., 2000); Tucker e colaboradores (2002) analisaram o óleo de $M$. coquimbensis, nativa do Chile, e identificaram como principais produtos limoneno, $\alpha$-pineno e carvona. M. pseudomato, da Argentina, foi caracterizado pela presença de 1,8-cineol e $\beta$-cariofileno (Demo, et al., 2003), enquanto que esta mesma espécie coletada na Bolívia apresentou 1,8-cineol, $\alpha$-pineno e linalol como compostos majoritários (Arze et al., 2005). Outra espécie, nativa da Bolívia, M. osteomeloides, teve seu óleo analisado e caracterizado pela presença de 1,8-cineol e $\alpha$-pineno (Arze et al., 2005). O óleo de M. rhopaloides, coletada no Equador, foi caracterizado pela presença predominante de geranial (Malagon et al., 2003). O óleo volátil de M. cisplatensis da Argentina foi caracterizado por 1,8-cineol e limoneno como componentes majoritários (Taher et al., 1983; Zygadlo et al., 1997), enquanto que o óleo da mesma espécie, porém coletada no Uruguai, apresentou 1,8-cineol e $\alpha$ pineno como principais produtos (Lorenzo et al., 2001). Para o óleo de $M$. pungens, proveniente da Argentina, foi relatado a presença majoritária de 1,8-cineol e limoneno (Zygadlo et al., 1997; Lorenzo et al., 2001; Ubiergo et al., 1986).

Como parte de projeto de investigação sistemática de óleos voláteis de espécies da família Myrtaceae, este trabalho aborda a análise da composição dos óleos obtidos por hidrodestilação de folhas frescas de M. cisplatensis, M. gigantea e M. pungens coletadas de populações nativas do Rio Grande do Sul. Até o presente momento não há estudos da composição química do óleo de $M$. gigantea.

\section{MATERIAL E MÉTODOS}

\section{Material vegetal}

Folhas de $M$. cisplatensis, $M$. gigantea e $M$. pungens foram coletadas de populações nativas no Rio Grande do Sul. Todas as espécies foram obtidas durante a fase estéril. Os exemplares das espécies foram identificados por M. Sobral e depositadas no Herbário do Departamento de Botânica da UFRGS (ICN). O local de coleta e os números de registro no herbário estão apresentados na Tabela 1 .

\section{Obtenção do óleo volátil}

O óleo volátil foi obtido a partir das folhas frescas, reduzidas com auxílio de triturador mecânico e submetidas à hidrodestilação em processo contínuo utilizando aparelho Clevenger, durante $4 \mathrm{~h}$ (Farmacopéia, 1988; OMS, 1992).

\section{Análise química}

Para a análise, os óleos voláteis obtidos por hidrodestilação foram diluídos em éter etílico na razão de 2:100 (v/v). O material diluído foi quantificado por cromatografia gasosa capilar equipada com detector de ionização de chamas (CG/DIC) e identificado por cromatografia gasosa capilar acoplada à espectrometria de massas (CG/EM).

Os óleos diluídos foram quantificados em um cromatógrafo gasoso Shimadzu GC 17A equipado com coluna capilar de sílica fundida Durabond-DB (30 $_{5} \mathrm{~m}$ $0,25 \mathrm{~mm} \times 0,25 \mu \mathrm{m})$ para a separação dos constituintes. A temperatura do injetor e detector foi de $220^{\circ} \mathrm{C}$ e 250 ${ }^{\circ} \mathrm{C}$, respectivamente, e a programação de temperatura foi de 60 a $300{ }^{\circ} \mathrm{C}$ a $3{ }^{\circ} \mathrm{C} / \mathrm{min}$, utilizando hélio como gás de arraste a uma pressão de $80 \mathrm{Kpa}$ e velocidade linear de $1 \mathrm{~mL}$ por minuto. A composição percentual foi obtida por integração eletrônica utilizando software CR10 (Shimadzu), pela técnica de normalização.

A análise qualitativa foi realizada utilizando-se o mesmo equipamento, nas mesmas condições, porém acoplado a um espectrômetro de massas utilizando aparelho Shimadzu QP5000 com quadrupolo cilíndrico, operando com energia de ionização de $70 \mathrm{eV}$ e temperatura de interface de $250^{\circ} \mathrm{C}$.

A identificação dos componentes do óleo foi baseada no índice de retenção linear (Índice de Kovats)

Tabela 1. Dados de coleta e rendimento percentual em óleo volátil de folhas das espécies de Myrcianthes do Rio Grande do Sul.

\begin{tabular}{|llllc}
\hline Espécie & ICN & Local de coleta & Data & Rendimento (\%) \\
\hline M. cisplatensis & Sobral 9226 & Alegrete & $14 / 03 / 01$ & 0,2 \\
M. gigantea & Sobral 8675 & Espumoso & $30 / 08 / 98$ & 0,1 \\
M. pungens & Sobral 8609 & Viamão & $10 / 04 / 97$ & 0,1 \\
\hline
\end{tabular}


Tabela 2. Composição percentual do óleo volátil das folhas de espécies de Myrcianthes do Rio Grande do Sul.

\begin{tabular}{|c|c|c|c|c|}
\hline Constituintes & $\mathrm{IK}^{*}$ & M. cisplatensis & M. gigantea & M. pungens \\
\hline \multicolumn{2}{|c|}{ Monoterpenos hidrocarbonados } & 20,8 & 11,5 & 5,9 \\
\hline$\alpha$-Pineno & 926 & 8,9 & 3,5 & 2,1 \\
\hline$\beta$-Pineno & 974 & 0,3 & 2,8 & 0,8 \\
\hline$\beta$-Mirceno & 980 & 0,4 & - & 0,3 \\
\hline$p$-Cimeno & 1015 & - & 0,7 & 0,4 \\
\hline Limoneno & 1021 & 10,9 & 4,5 & 1,9 \\
\hline$\gamma$-Terpineno & 1034 & 0,3 & - & - \\
\hline (E)- $\beta$-Ocimeno & 1037 & - & - & 0,4 \\
\hline \multicolumn{2}{|c|}{ Monoterpenos oxigenados } & 35,5 & 5,5 & 5,0 \\
\hline 1,8-Cineol & 1024 & 29,8 & 1,4 & 2,7 \\
\hline Linalol & 1092 & - & 0,7 & 0,5 \\
\hline (E)-Pinocarveol & 1130 & - & 0,9 & - \\
\hline Terpinen-4-ol & 1166 & - & 0,4 & 0,8 \\
\hline$\alpha$-Terpineol & 1180 & 5,7 & 1,2 & 1,0 \\
\hline$(E)$-Carveol & 1210 & - & 0,5 & - \\
\hline Carvotanacetona & 1233 & - & 0,4 & - \\
\hline \multicolumn{2}{|c|}{ Sesquiterpenos hidrocarbonados } & 20,2 & 11,1 & 45,4 \\
\hline$\alpha$-Copaeno & 1363 & - & 2,6 & - \\
\hline$\beta$-Elemeno & 1387 & - & 1,6 & 9,1 \\
\hline$\beta$-Cariofileno & 1414 & 10,8 & 1,2 & 10,1 \\
\hline Aromadendreno & 1434 & 2,5 & - & 1,1 \\
\hline$\alpha$-Humuleno & 1444 & 2,0 & - & 2,0 \\
\hline allo-Aromadendreno & 1456 & 0,6 & 1,9 & 1,3 \\
\hline$\gamma$-Gurjuneno & 1463 & - & - & 0,6 \\
\hline$\gamma$-Muuroleno & 1467 & - & - & 0,8 \\
\hline$\beta$-Selineno & 1478 & 0,8 & 2,5 & 3,1 \\
\hline$\alpha$-Selineno & 1480 & 2,7 & - & - \\
\hline$\alpha$-Muuroleno & 1487 & - & 0,6 & - \\
\hline Bicyclogermacreno & 1492 & - & - & 6,9 \\
\hline Germacreno A & 1494 & - & - & 0,7 \\
\hline$\beta$-Bisaboleno & 1500 & - & - & 3,3 \\
\hline$\gamma$-Cadineno & 1504 & - & 0,7 & - \\
\hline$\delta$-Cadineno & 1514 & 0,8 & - & 2,0 \\
\hline$(E)-\gamma$-Bisaboleno & 1523 & - & - & 3,3 \\
\hline Germacreno B & 1552 & - & - & 1,1 \\
\hline \multicolumn{2}{|c|}{ Sesquiterpenos oxigenados } & 19,8 & 58,5 & 37,4 \\
\hline Espatulenol & 1573 & 0,8 & 28,9 & 9,7 \\
\hline Óxido de cariofileno & 1575 & - & 6,7 & 2,3 \\
\hline Globulol & 1580 & 4,8 & 2,8 & 6,2 \\
\hline epi-Globulol & 1588 & 1,2 & - & 4,7 \\
\hline Guaiol & 1590 & 4,9 & - & - \\
\hline 5-epi-7-epi-Eudesmol & 1595 & 1,0 & - & - \\
\hline Eudesmol ${ }^{* *}$ & 1600 & 1,3 & - & - \\
\hline iso-Espatulenol & 1603 & - & 9,5 & - \\
\hline Óxido de humulene I & 1607 & - & - & - \\
\hline 10-epi- $\gamma$-Eudesmol & 1608 & - & - & 1,9 \\
\hline 1-epi-Cubenol & 1623 & 1,2 & 1,3 & - \\
\hline$\tau$-Muurolol & 1639 & - & 1,3 & 2,1 \\
\hline$\gamma$-Eudesmol & 1628 & 0,9 & - & - \\
\hline$\alpha$-Muurolol & 1641 & - & 1,0 & - \\
\hline$\beta$-Eudesmol & 1643 & 1,6 & - & - \\
\hline$\alpha$-Eudesmol & 1645 & 1,8 & - & - \\
\hline \multirow[t]{2}{*}{$\alpha$-Cadinol } & 1647 & - & 7,0 & 8,0 \\
\hline & & & & $\begin{array}{r}\text { Rev. Bras. Farmacogn. } \\
\text { Braz J. Pharmacogn. } \\
\text { 16(3):jul/set. } 2006\end{array}$ \\
\hline
\end{tabular}




\begin{tabular}{lllll}
$\beta$-Bisabolol & 1659 & - & - & 1,0 \\
$\alpha$-Bisabolol & 1673 & - & - & 0,9 \\
$\tau$-Bisabolol & 1675 & - & - & 0,6 \\
Bulnesol & 1652 & 0,3 & - & - \\
& & & $\mathbf{3 , 5}$ \\
Outros & & $\mathbf{1 , 5}$ & - & $\mathbf{0 , 5}$ \\
Acetato de etila & 791 & 1,4 & 0,3 & - \\
Hexenal & 798 & - & - & 0,2 \\
$(E)-3$-Hexenol & 838 & - & 0,3 & - \\
$(E)-$-Hexenal & 842 & 0,1 & 2,4 & - \\
$(Z)-3-H e x e n o l$ & 846 & - & 0,5 & 0,3 \\
$n$-Hexanol & 858 & - & $\mathbf{9 0 , 1}$ & $\mathbf{9 4 , 2}$ \\
\hline Total & & $\mathbf{9 7 , 8}$ &
\end{tabular}

* IK: Índice de retenção linear em coluna DB5. ** Isômero não identificado.

calculado em relação aos tempos de retenção de uma série homóloga de $n$-alcanos e no padrão de fragmentação observado nos espectros, por comparação com amostras autênticas, dados retirados da literatura (Adams, 2001; Apel et al., 2002; Apel et al., 2004a; Apel et al., 2004b; Apel et al., 2005; Agostini et al., 2005) ou, ainda, por comparação com espectroteca própria e NIST62.

\section{RESULTADOS E DISCUSSÃO}

A análise da composição química dos óleos voláteis das espécies de Myrcianthes está apresentada na Tabela 2. Todas as três espécies apresentaram rendimento entre 0,1 e $0,2 \%$. Foi identificado um total de 59 compostos.

Para o óleo de $M$. gigantea foi possível identificar 30 compostos, representando $90,1 \%$ do teor de voláteis. Este óleo foi caracterizado pela predominância de sesquiterpenos oxigenados, em especial do grupo germacrano, cujo composto majoritário foi o espatulenol (28,9\%) seguido, em menor quantidade, de seu isômero, iso-espatulenol $(9,5 \%)$. Para esta espécie não há relatos de estudos realizados com o óleo volátil.

O óleo de $M$. cisplatensis foi caracterizado pela predominância de monoterpenos oxigenados (35,5\%), em especial 1,8-cineol (29,8\%), seguido pelos hidrocarbonetos monoterpênicos $(20,8 \%)$ limoneno $(10,9 \%)$ e $\alpha$-pineno (8,9\%). Também é observado quantidade apreciável de $\beta$-cariofileno $(10,8 \%)$, um hidrocarboneto sesquiterpênico do grupo dos cariofilanos. Para esta espécie foram identificados 27 componentes totalizando $97,8 \%$ do óleo.

O óleo volátil de $M$. cisplatensis, apresentou como constituinte majoritário o monoterpeno 1,8-cineol com teor de $29,8 \%$. Relatos anteriores para a espécie indicam também este componente como majoritário, com teores de $40,7 \%, 13,5 \%$ e $53,8 \%$ para exemplares coletados em dois locais diferentes na Argentina (Taher et al., 1983; Zygadlo et al., 1997) e no Uruguai (Lorenzo et al., 2001), respectivamente. Limoneno foi encontrado em teor de $10,9 \%$, enquanto os relatos da literatura indicaram $22,1 \%, 6,5 \%$ para as espécies da Argentina (Taher et al., 1983; Zygadlo et al., 1997). A espécie coletada no Uruguai apresentou também teor de $\alpha$-pineno (16,6\%) mais elevado que a amostra do Brasil (8,9\%) (Lorenzo et al., 2001).

O óleo volátil de $M$. pungens foi o que apresentou maior complexidade, sendo identificado 36 constituintes, nenhum em quantidade significativa. Dentre as frações, os hidrocarbonetos sesquiterpênicos foram os de maior percentagem $(45,4 \%)$, onde o principal composto foi o $\beta$-cariofileno $(10,1 \%)$, seguido por $\beta$-elemeno $(9,1 \%)$. Estudos com o óleo desta espécie obtido a partir de duas coletas realizadas na Argentina, demonstraram a presença majoritária dos monoterpenos 1,8-cineol e limoneno (13,5\% e $17,3 \%$, respectivamente) para uma das análises, e para a outra, 1,8-cineol $(45,8 \%)$, pulegona $(9,4 \%)$ e farnesol $(9,0 \%)$ foram os principais componentes do óleo, sendo estes dois últimos ausentes na primeira análise (Zygadlo et al., 1997; Ubiergo et al., 1986). No presente trabalho, a fração monoterpênica não se mostrou relevante.

\section{CONCLUSÕES}

Todas as espécies analisadas apresentaram uma composição química bastante complexa, em especial para $M$. pungens. Os óleos obtidos de $M$. gigantea e $M$. pungens foram caracterizados pela predominância de sesquiterpenos cíclicos, principalmente os derivados das vias de ciclização do cariofilano, germacrano e cadinano, enquanto que $M$. cisplatensis apresentou, principalmente, substâncias das séries $p$-mentano e pinano. Pode-se, ainda, observar para $M$. pungens, a possível ocorrência de quimiotipos, uma vez que estudos realizados revelaram predominância de monoterpenos e a espécie aqui estudada apresentou destaque para sesquiterpenos. O óleo de $M$. cisplatensis apresenta composição similar à relatada na literatura, com caracterização do óleo por monoterpenos, em especial 1,8-cineol e limoneno (Zygadlo et al., 1997; Lorenzo et al., 2001), o que parece ser uma constância para esta espécie.

O conteúdo do óleo, em uma planta, pode variar em função da época do ano e do seu estádio de 
desenvolvimento (Goralka et al., 1996; Dunlop et al., 2000; Sousa et al., 2005; Carvalho-Filho et al., 2006). Fatores geográficos (localização) e ecológicos (habitat) também devem ser considerados (Oliveira et al., 2005). Além de todos estes parâmetros deve-se levar em conta a variabilidade genética das plantas, que está intimamente relacionada com a qualidade dos óleos voláteis, e é expressa através dos chamados quimiotipos (Tavares et al., 2005). A variação química ocorre com predomínio de diferentes compostos no óleo volátil obtido a partir de uma mesma espécie coletada em locais diferentes, o que é freqüente devido à alta complexidade química dos óleos voláteis.

\section{AGRADECIMENTOS}

Este trabalho contou com auxílio financeiro e bolsas de estudo do CNPq, CAPES e FAPERGS.

\section{REFERÊNCIAS}

Adams RP 2001. Identification of essential oil components by gas chromatography quadrupole mass spectrometry. Allured Publishing Corp., Carol Stream, IL.

Agostini F, Santos ACA, Rossato M, Pansera MR, Zattera F, Wasum R, Serafini LA 2005. Estudo do óleo essencial de algumas espécies do gênero Baccharis (Asteraceae) do sul do Brasil. Rev Bras Farmacogn 15: 215-219.

Apel MA, Limberger RP, Sobral M, Ntalani H, Verin P, Menut C, Bessiere JM, Henriques AT 2002. Chemical composition of the essential oils from southern Brazilian Eugenia species. Part III. J Essent Oil Res 14: 259-262.

Apel MA, Sobral M, Schapoval EES, Menut C, Bessiere JM, Henriques AT 2004a. Chemical composition of the essential oils of Eugenia hyemalis and Eugenia stigmatosa. Part VI: section biflorae. $J$ Essent Oil Res 16: 437-439.

Apel MA, Sobral M, Schapoval EES, Menut C, Bessiere JM, Henriques AT 2004b. Chemical composition of the essential oils of Eugenia beaurepaireana and Eugenia pyriformis: Section dichotomae. $J$ Essent Oil Res 16: 191-192.

Apel MA, Sobral M, Menut C, Bassiere JM, Zuanazzi JA, Schapoval EES, Henriques AT 2005. Volatile constituents of four Hexachlamys species growing in South Brazil. Flavour Frag J 20: 176-179.

Arze JBL, Jean FI, Gagnon H, Collin G, Garneau F, Pichette A 2005. Essential oils from Bolivia. VIl. Myrtaceae: Myrcianthes osteomeloides (Rusby) McVaugh and Myrcianthes pseudomato (Legrand) McVaugh. J Essent Oil Res 17: 64-65.

Carvalho-Filho JLS, Blank AF, Alves PB, Ehlert PAD, Melo AS, Cavalcanti SCH, Arrigoni-Blank MF, SilvaMann RS 2006. Influence of the harvesting time, temperature and drying period on basil (Ocimum basilicum L.) essential oil. Rev Bras Farmacogn 16: $24-30$

Demo MS, Oliva MM, Zunino MR, Lopez ML, Zygadlo JA 2003. Aromatic plants from Yungas. Part
IV: composition and antimicrobial activity of Myrcianthes pseudomato essential oil. Pharma Biol 40: 481-484.

Carmen R, Torre C, Retamar JA 1972. Essential oil of Myrcianthes callicoma. Essenze Deriv Agrum 42: 429-432.

Dunlop PJ, Bignell CM, Hibbert DB 2000. Use of gas chromatograms of essential leaf oils to compare clones of Eucalyptus camaldulensis. Biochem Syst Ecol 28: 383-391.

Farmacopéia Brasileira 1988. 4 ed. Parte II, São Paulo: Ateneu, p.v.4.2.6.

Font Quer P 1988. Plantas medicinales. Barcelona: El Dioscórides Renovado.

Goralka JLR, Schumaker MA, Langenheim JH 1996. Variation in chemical and physical properties during leaf development in California Bay tree (Umbellularia californica): predictions regarding palatability for deer. Biochem Syst Ecol 24: 93-103.

Grifo FT 1992. A revision of Myrcianthes Berg (Myrtaceae). New York. Doctoral Dissertation - Cornell University.

Kott V, Barbini L, Cruañes M, Muñoz JD, Vivot E, Cruañes J, Martino V, Ferraro G, Cavallaro L, Campos R 1999. Antiviral activity in Argentine medicinal plants. $J$ Ethnopharmacol 64: 79-84.

Lorenzo D, Dellacassa E, Bonaccorsi I, Mondello L 2001. Uruguayan essential oils. Composition of leaf oil of Myrcianthes cisplatensis (Camb.) Berg. ('Guayabo colorado') (Myrtaceae). Flavour Frag J 16: 97-99.

Malagon O, Vila R, Iglesias J, Zaragoza T, Caniguera S 2003. Composition of the essential oils of four medicinal plants from Ecuador. Flavour Frag J 18: 527-531.

Marchiori JNC, Sobral M 1997. Dendrologia das Angiospermas - Myrtales. Santa Maria: Ed. da UFSM.

Oliveira RN, Dias IJM, Câmara CAG 2005. Estudo comparativo do óleo essencial de Eugenia punicifolia (HBK) DC. de diferentes localidades de Pernambuco. Rev Bras Farmacogn 15: 39-43.

OMS - Quality control methods for medicinal plant materials 1992, Geneve: Organisation Mondiale de La Sante.

Penna C, Marino S, Vivot E, Cruañes MC, Muñoz JD, Crúañes J, Ferraro G, Gutkind G, Martino V 2001. Antimicrobial activity of Argentine plants used in the treatment of infectious diseases. Isolation of active compounds from Sebastiania brasiliensis. $J$ Ethnopharmacol 77: 37-40.

Pino JA, Rosado A, Bello A, Urquiola A, Garcia G 2000. Essential oil of Myrcianthes fragrans (Sw.) McVaugh from Cuba. J Essent Oil Res 12: 225-226.

Pio Correa M 1984. Dicionário das plantas úteis do Brasil e das exóticas cultivadas, Rio de Janeiro: Imprensa Nacional, vol. III.

Sousa LA, Albuquerque JCR, Leite MN, Stefanini MB 2005. Sazonalidade dos ductos secretores e óleo essencial de Foeniculum vulgare var. vulgare Mill. (Apiaceae). Rev Bras Farmacogn 15: 155-161.

Taher HA, Santi de Bongioanni MN, Talenti ECJ 1983. Study of the Essential Oil of Myrcianthes cisplatensis (Cambassedes) Berg. Essenze Deriv Agrum 53: 1325.

Tavares E.S, Julião LS, Lopes D, Bizzo HR, Lage CLS, Leitão SG 2005. Análise do óleo essencial de folhas de três quimiotipos de Lippia alba (Mill.) 
N.E.Br. (Verbenaceae) cultivados em condições semelhantes. Rev Bras Farmacogn 15: 1-5.

Tucker AO, Maciarello MJ, Landrum LR 1992. Volatile leaf oils of Caribbean Myrtaceae. VI. Myrcianthes fragrans (S W.) Mc Vaugh of Jamaica. J Essent Oil Res 4: 313-314.

Tucker AO, Maciarello MJ, Landrum LR 2002. Volatile leaf oil of Myrcianthes coquimbensis (Barneoud) Landrum et Grifo (Myrtaceae) of Chile. J Essent Oil Res 14: 40-41.

Ubiergo G, Taher HA, Talenti EC 1986. Mono and sesquiterpenoids from the essential oil of Myrcianthes pungens. An Asoc Quim Argent 74: 567-9.

Zygadlo JA, Rotman AD, Alonso MJP, Negueruela AV 1997. Leaf oils of two Myrcianthes species from Argentina: M. pungens (Berg.) Legrand and M. cisplatensis (Camb.) Berg. J Essent Oil Res 9: 237-239. 